\title{
Von Wissen und Wahn, Romanen und Rittern: (Un)nützes Wissen als Don Quixoterie?
}

Scheinbar Unnützes Wissen ganz unterschiedlicher Art findet sich nicht zu sparsam in Cervantes' Roman Don Quixote von La Mancha (1605/1615). ${ }^{1}$ Wenn etwa über beträchtliche Passagen hinweg geschildert wird, wie Sancho Pansa mitten in der Nacht sein Geschäft verrichten kann, ohne vom Esel zu steigen, ${ }^{2}$ fragt man sich als Leser, wie viel der Information tatsächlich nötig gewesen wäre. Oder, wenn fast jedes einzelne Buch in Don Quixotes Bibliothek aufgezählt, literaturkritisch beäugt und kommentiert wird, erwischt man sich schon mal beim Überblättern einiger Seiten. Insgesamt spielt Wissen in unterschiedlichen Schattierungen eine Rolle in diesem Roman. ${ }^{3}$ Ins-

1 Miguel de Cervantes Saavedra, Leben und Taten des scharfsinnigen Edlen Don Quixote von La Mancha. Aus dem Spanischen von Ludwig Tieck. Mit einem Essay von Heinrich Heine und Illustrationen von Gustave Doré, Zürich 1987 (Titel im spanischen Original: $\mathrm{El}$ ingenioso hidalgo Don Quixote de la Mancha). Im Folgenden zitiert unter der Sigle DQ.

2 Vgl. DQ, Teil I, Kapitel 20. Apropos Unnützes Wissen: In der katalanischen Tradition existiert eine unübliche Krippenfigur, die an diese Szene erinnert und die es auch in Form von Don Quixote und Sancho Panza gibt, nämlich die des scaganer` (von cagar: seine Notdurft verrichten`). Darunter ist ein hockendes Männchen zu verstehen, das mit bis an die Knöchel heruntergelassener Hose sein Geschäft verrichtet. Die Figur wird in der Krippe - aus 'Respekt ‘ für den Heiland - an einem zunächst weniger auffälligen Örtchen aufgestellt (Kinder machen sich daraus den weihnachtlichen Spaß, diese Figur zu suchen - etwa wie mit der amerikanischen schristmas pickle im Weihnachtsbaum). Die Figur ist ein Zeichen für Glück und Fruchtbarkeit - und hält mittlerweile Einzug in Polit- und Gesellschaftssatire, denn die Figuren verkaufen sich besonders gut, wenn sie internationale Politiker oder andere 'Prominente darstellen: "Among the international figures, President Nicolas Sarkozy, Silvio Berlusconi and the Pope are all strong sellers; Spiderman and Sponge Bob do well«, vgl. Sarah Rainsford, A traditional Nativity scene, Catalan-style, BBC, 23.12.2010, http://www.bbc.co.uk/news/world-europe-12059969 [Datum des letzten Zugriffs: 08.09.2014].

3 Es werden zahlreiche Themen, Wissensbereiche und Diskurse angeschnitten: unter anderem etwa kulturelles Wissen über Soziolekte und unterschiedliche Gesellschaftsmilieus, Diskurse über Kirche, Inquisition und Glaubensrichtungen (auch die Problematik der conversos), der Status der Frau in der Gesellschaft, die Problematik des Sklavenhandels, die Frage nach Gerechtigkeit, die Bedeutung der Wissenschaft und damit zusammenhängend auch Reflexionen über Technik und deren Entwicklung. Es wird beispielsweise über den Gebrauch von Feuerwaffen, über Apparate wie die Druckerpresse, Spektakel wie Feuerwerke, etc. räsoniert: "Cervantes' bekanntester Roman erzählt von einer Welt voller technischer Neuerungen, die den Helden immer wieder beschäftigen und teilweise auch 
besondere stellt sich die Frage nach dem Nutzen bzw. Un-nutz literarischen Wissens für die Literatur selbst. Im Folgenden soll also nicht die Rede davon sein, wie sich sliteraturfremder Wissens- und Wissenschaftsdiskurse in der Literatur niederschlagen. Vielmehr soll gezeigt werden, inwiefern in Don Quixote ein literarischer Wissensdiskurs inszeniert wird, bei dem sich die Literatur selbst zum Wissen wird, sich als solche befragt, ihren eigenen Status und ihre Möglichkeiten auslotet und sich nicht zuletzt auch selbst ironisiert und parodiert - wie also eine metapoetische Reflexion der Literatur über ihr Wissensverhältnis zu sich selbst stattfindet. In Don Quixote werden hinsichtlich literarischen Wissens oftmals absurde und skurrile Handlungs- und Erzählbewegungen entworfen, von Wissensdifferenzen geprägte narrative Situationen und Figurenkonstellationen inszeniert; es wird ein heterogenes Nebeneinander von Themen, Motiven, Genres und intertextuellen Bezügen gestaltet: Der Text scheint sich insgesamt durch eine - übrigens auch oft verwirrende - Ästhetik und Poetik der Redundanz und scheinbaren Irrelevanz auszuzeichnen. Dadurch wird literarisches Wissen an der Oberfläche als unnütz markiert. Es wird daher herauszufinden sein, ob es sich bei dieser Struktur um eine sogenannte 'Don Quixoterie handelt, also um ein Moment, das genauso absurd und scheinbar unnütz ist wie Don Quixotes von Verirrung und Verwirrung geprägte Unternehmungen, die oftmals in der Situation des Scheiterns enden - wie etwa sein berühmter Kampf gegen Windmühlen -, oder ob dieser Poetik und Ästhetik des Scheiterns und des unnützen literarischen Wissens ein funktionales Potential innewohnt.

\section{Literarisches Wissen und Wahn, Romane und Ritter}

Miguel de Cervantes' Roman Don Quixote von La Mancha erscheint im spanischen Original 1605 (erster Teil) und 1615 (zweiter Teil) und erzählt, dem Untertitel zufolge, die "Leben und Taten" eines "scharfsinnigen Edlen«. Hinter diesem glorreichen wie ironischen Titel versteckt sich bekanntermaßen die Geschichte des Alonso Quixano, der seinen Namen in Don Quixote von La Mancha umdichtet und daraufhin als irrender Ritter auf seinem unterernährten Pferd Rosinante in die spanische Welt ausreitet, denn er fühlt sich gerufen vom »Unrecht, das zu vertilgen, [der] Ungebühr,

sein Handeln bestimmen«, siehe: Wolfram Nitsch, Der hohle Kopf. Don Quijote und die Technik, in: Christoph Strosetzki (Hg.), Miguel de Cervantes' Don Quijote. Explizite und implizite Diskurse im Don Quijote, Berlin 2005, S. 137-148, hier S. 138. 
die er einrichten, [der] Beschwer, die er aufheben, [den] Mißbräuche[n], die er bessern und [den] Verschuldungen, die er vergelten müsse« (DQ 29). Seine Absichten sind dabei zwar immer edel und hehr, doch das Problem bei seinem Unterfangen ist, dass zu seiner Lebenszeit bereits längst keine fahrende Ritterschaft mehr existiert. Das noch größere Problem ist, dass der Grund für seine Ausritte dem Verlust seines Verstandes zuzuschreiben ist. Letzteren büßt er durch die exzessive Lektüre von Ritterromanen ein. Diese Lektüre von sunnützer Literatur lässt ihn nützliche, weil lebenserhaltende, Beschäftigungen vergessen:

Es ist zu wissen, daß obgenannter Edler die Zeit, die ihm zur Muße blieb (und dies betrug den größten Teil des Jahres), dazu anwandte, Bücher von Rittersachen mit solcher Liebe und Hingebung zu lesen, daß er darüber sowohl die Ausübung der Jagd als auch die Verwaltung seines Vermögens vergaß [...]. Sein Lesen also verwickelte ihn so, daß er die Nächte damit zubrachte, weiter und weiter, und die Tage sich tiefer und tiefer hineinzulesen; und so kam es vom wenigen Schlafen und vielem Lesen, daß sein Gehirn ausgetrocknet wurde, wodurch er den Verstand verlor [...]. Er bildete dabei sich fest ein, daß alle diese erträumten Hirngespinste, die er las, wahr wären, daß es für ihn auf der Welt keine zuverlässigere Geschichte gab. (DQ25 u. 26)

Don Quixote glaubt an die fiktionalisierten, literarisch verbrämten und rhetorisch ästhetisierten Rittergeschichten, ${ }^{4}$ als seien sie Realität, und versucht, sie in Lebenspraxis umzuwandeln. Ein Verhalten, das sich übrigens auch bei Flauberts Figuren Bouvard und Pécuchet in Bezug auf Theorie beobachten lässt, durch das sich beide durchaus als Don Quixote-Figuren interpretieren lassen. ${ }^{5}$ Jedenfalls bestimmen diese Rittergeschichten Don Quixotes Wahrnehmung und dienen als Modell der Nachahmung und damit als Leitfaden seiner Handlungen - so ist sein "gewöhnliches Mittel [...] an irgendeine Stelle in seinen Büchern zu denken« (DQ 47). Die Folge davon: Windmühlen nimmt er als Riesen, Schafherden als feindliche Soldatenheere wahr; überhaupt glaubt er insgesamt, die Welt würde vom Willen eines

4 Der Text inszeniert die mangelnde Qualität der von Don Quixote so geliebten Romane durch die Darstellung einer maßlos übertriebenen `Ästhetisierung`, die an die totale Sinnlosigkeit reicht, z.B.: "Das Tiefsinnige des Unsinnlichen, das meinen Sinnen sich darbeut, erschüttert also meinen Sinn, daß ich über Eure Schönheit eine vielsinnige Klage führe« (DQ25f).

5 Vgl. Alexander Welsh, The Influence of Cervantes, in: Anthony Cascardi (Hg.), The Cambridge Companion To Cervantes, Cambridge 2002, S. 80-98 und Howard Mancing, The Cervantes Encyclopedia, Bd. I, Westport CT 2004, S. 297. Siehe dazu auch den Beitrag von Stefan Willer in diesem Band. 
großen Magiers namens Freston bestimmt, der ihm feindlich gesonnen sei. ${ }^{6}$ Sein Verstandesverlust führt zum Verkennen der ontologischen Differenz von Literatur und Wirklichkeit, alles steht für ihn im Zusammenhang der epischen Wunderwelt der Ritterbücher. Der blinde Aktionismus, ${ }^{7}$ den er dabei an den Tag legt, stellt im Prinzip nichts anderes als den Versuch dar, der Wirklichkeit sein literarisches Wissen aufzuzwingen und sie in "gelebte Literatur ${ }^{8}$ zu verwandeln. Daraus resultiert denn auch die stete Struktur des Scheiterns seiner Unternehmungen, die sich teils in profanen Schlägereien - Don Quixote und Sancho werden regelmäßig verprügelt - oder dem absoluten Nicht-Verständnis von Seiten anderer Figuren äußert, denn »[...] die prosaische Welt ist und bleibt die stärkere. Die wirkliche Beschaffenheit der Welt beweist ihre Resistenz und setzt sich unablässig gegen die Illusion ${ }^{9}$ von Don Quixote durch.

II. Wissensdifferenz in den Figurenkonstellationen: Unpraktisches` Wissen als Unnützes Wissen?

Die irritierten Reaktionen der Umwelt auf den selbsternannten Ritter und das 'Zurückschlagen der Wirklichkeit in Form von Handgreiflichkeiten seitens anderer Figuren zeigt ein Wissensgefälle zwischen Don Quixote und seiner sozialen Umgebung an. In Don Quixote selbst stellt der Wahn ja eine Schieflage von Wissen dar: Sein literarisches Wissen bildet einen >Wissensüberschuss`, der fast alles andere überschattet. Dieses literarische Wis-

6 mDieser ist ein weiser Zauberer und mein großer Feind, denn er ist mir grämlich, [...] [er] erzeigt [...] mir so viele Unart, als er nur kann«, DQ 59.

7 In der Interpretation von Georg Lukács handelt es sich dabei um »[d]as vollständige Fehlen einer innerlich erlebten Problematik", das "die Seele in reine Aktivität [verwandelt]. Weil sie in ihrem essentiellen Sein von allem unberührt in sich ruht, muß jede ihrer Regungen eine Handlung nach außen sein. Das Leben eines solchen Menschen muß also zu einer ununterbrochenen Reihe selbstgewählter Abenteuer werden«, siehe: Georg Lukács, Die Theorie des Romans. Ein geschichtsphilosophischer Versuch über die Formen der großen Epik, München ${ }^{2} 2000$, S. 85.

8 Theodor Wolpers (Hg.), Gelebte Literatur in der Literatur. Studien zu Erscheinungsformen und Geschichte eines literarischen Motivs. Bericht über Kolloquien der Kommission für literaturwissenschaftliche Motiv- und Themenforschung 1983-1985, Göttingen 1986.

9 Heinz-Peter Endress, Don Quijotes Ideale im Umbruch der Werte vom Mittelalter bis zum Barock, Tübingen 1991, S. 18. Georg Lukács spricht in diesem Zusammenhang, in Anschluss an Hegel, von der "prosaische[n] Niedertracht« der Umstände, siehe: Georg Lukács, Die Theorie des Romans, S. 90. 
sen besitzen die anderen Figuren meist nicht, woraus denn auch die kaum überbrückbare Wissensdisjunktion zwischen diesen und Don Quixote resultiert. Es entstehen daher oftmals Kommunikationsprobleme, wie sich beispielsweise in der Kastellszene zeigt: Don Quixote wird auf seinem ersten Auszug von - seiner Wahrnehmung zufolge - holden Damen und einem Kastellan zum Nachtessen in ein Kastell eingeladen. Um seine Huldigung zu erweisen, beginnt er spontan nach der Art der Rittergeschichten in Versen zu sprechen: "Niemals ward ein edler Bote/So bedient von Damen süß,/ Wie der große Don Quixote,/Als er seine Heimat ließ« (DQ33). Allerdings handelt es sich nicht um Damen, sondern um Freudenmädchen, bei dem Kastell um ein Freudenhaus. Den Freudenmädchen fehlt natürlich das nötige literarische und rhetorische Wissen, um Don Quixotes elaborierte Rede zu verstehen: "Diese Sprache verstanden die Damen nicht [...] sie konnten das Lachen nicht zurückhalten« (DQ 32) und "Die Mädchen, die solcher rhetorischen Figuren ungewohnt waren, antworteten nicht darauf [...]« (DQ 34). Ein tatsächlicher Austausch mit ihm wird erst möglich, wenn das Gegenüber seinen Wahn erkennt, seinen Code realisiert, diesen verwendet und sein Verhalten und seine Rede in den Wissenskontext des Ritterromans einpasst - wie es der 'Kastellan` dann im Folgenden auch tut. Übrigens kommt dadurch, dass die Erzählinstanz stets eine »deutliche Differenzierung [herstellt] zwischen figuraler Wahnperspektive [des Don Quixote]« und eines glaubhaften Diskurses der Vernunft, der eine "Koalition« mit den "vernünftigen Figuren« innerhalb des Romans bildet, »für den Leser nicht der geringste Zweifel auf[...] «, ${ }^{10}$ wo sich die Wahrheit der Situation ansiedelt. Der Leser wird damit zum Zuschauer der Wissensdifferenz zwischen Don Quixote und dessen Umwelt gemacht - wodurch für ihn der Wahn Don Quixotes markiert wird. Die Tatsache, dass Don Quixotes Weltsicht so gar nicht vereinbar ist mit seiner Umwelt, wirft natürlich die Frage auf, ob sein literarisches Wissen denn nicht eigentlich, zumindest was die praktische Lebenswelt angeht, ein tatsächlich Unnützes Wissen ist.

Vor allem die Figurenkonstellation der beiden Hauptfiguren ist geprägt von Wissensdifferenzen. Don Quixote wird als Figur auch durch die Gegenüberstellung mit seiner Satellitenfigur Sancho Pansa definiert, einem Bauern, den er als 'Stallmeister rekrutiert hat. Beide Figuren repräsentieren jeweils unterschiedliche Wissenspole. Während Don Quixote literarisches

10 Wolfgang Matzat, Die Welt des Don Quijote: Wirklichkeitskonstruktion und romanhistorischer Ort, in: Christoph Strosetzki (Hg.), Miguel de Cervantes' Don Quijote, S. 177-194, hier S. 180. 
Wissen - in gewisser Weise gelerntes und Gelehrtenwissen, insgesamt abstrakte Reflexion - repräsentiert, steht Sancho Pansa für praktisches und traditionell-volkstümliches Lebenswissen. Letzteres spiegelt sich beispielsweise in der hohen Frequenz der von Sancho verwendeten Sprichwörter - und Sprichwörter stellen im Prinzip kondensiertes Lebens- und Erfahrungswissen dar. ${ }^{11}$ Er repräsentiert aber auch Über-Lebenswissen, denn er zeichnet sich generell durch eine pragmatische Einstellung zur Wirklichkeit aus und weiß sich, wenn Don Quixote in seiner Literaturwelt schwebt, durchweg mit `Bauernschläue und bodenständigem Handeln aus verzwickten Situationen zu retten. Auch optisch wird diese Komplementäropposition ja deutlich gemacht: Don Quixote ist groß, dünn, geradezu ausgezehrt, während Sancho Pansa klein und rundlich ist. Überhaupt repräsentiert das Motiv der Körperlichkeit, des Essens und des leiblichen Wohls bzw. Un-Wohls den Unterschied zwischen den beiden Figuren und deren Wissenspolen. Durch Sancho, ausgerüstet mit Brotbeutel und Weinschlauch, ist stets für das Nötigste zum Überleben gesorgt, wohingegen Don Quixote der festen Überzeugung ist, Ritter würden bei ihren Auszügen nichts essen und nur von ihren Abenteuern und ihrer Minne leben. ${ }^{12}$ Dieses Wissen entnimmt er ex negativo den Ritterromanen, denn dort würde schließlich nie erwähnt, dass Ritter etwas äßen. ${ }^{13}$ Kümmert sich Don Quixote jedoch einmal im entferntesten Sinne um körperliches 'Wohk, wie im Falle eines von ihm gebrauten Heilbalsams für Ritter, kann das schon mal im kollektiven Erbre-

11 Dies wird treffend auf den Punkt gebracht, wenn es heißt: "'Man hat ein spanisches Sprichwort, das mir sehr wahr scheint, wie es denn alle sind, weil sie kurze Sentenzen enthalten, die aus einer langen und verständigen Erfahrung geschöpft sind [...] « (DQ 363). Während Sprichwörter einerseits "sprachliche Fertigware« darstellen können (vgl. Wolfgang Mieder, Andere Zeiten, andere Lehren. Sprichwörter zwischen Tradition und Innovation, Phraseologie und Parömiologie, Bd. 18, Hohengehren 2006, S. 26), zeugt die oft recht kreative Verwendung der Sprichwörter durch Sancho von dessen Gewitztheit. Des Öfteren setzt er sie strategisch zu seinen Zwecken ein, passt sie nach Belieben und Situation an, wandelt sie um, verzerrt sie.

12 „Don Quixote begehrte nicht zu frühstücken, weil er sich, wie schon gesagt, mit nahrhaften Vorstellungen unterhalten hatte« (DQ66). Also selbst das in den Romanen Nicht-Dargestellte sucht Don Quixote zu Handlungswissen für seine Wirklichkeit zu machen.

13 »[...] erfahre also, Sancho, daß die Ehre der irrenden Ritter darin besteht, in einem Monate nicht zu essen, und selbst wenn sie essen, das, was ihnen in die Hände fällt; du würdest auch davon versichert sein, wenn du so viele Historien wie ich gelesen hättest, denn trotz der großen Menge habe ich nicht in einer einzigen erwähnt gefunden, daß die irrenden Ritter gegessen hätten, wenn es sich nicht etwa traf, daß sie ein prächtiges Bankett anrichteten, sonst begnügten sie sich an den übrigen Tagen mit der Entbehrung [...]. Also, Freund Sancho, sorge nicht, mir etwas Schmackhaftes zu geben, wenn du nicht die Welt neu schaffen und die irrende Ritterschaft aus ihren Angeln heben willst« (DQ79). 
chen enden. ${ }^{14}$ Was Genuss oder einfach nur die schiere physische Existenzbewahrung angeht, erweist sich Sancho Pansas Lebenswissen als durchaus nützlich, weil lebenserhaltend, während Don Quixotes Wissen als unnütz und im Zweifelsfall gar als lebensgefährdend eingestuft werden muss. Die Situationen der Wissensdifferenzen tragen natürlich entscheidend zum humoristischen Charakter des Romans bei.

\section{III. Ästhetik der Irrelevanz}

Die Figurenkonstellationen in diesem Roman stellen auf vielfältige und spielerisch-burleske Weise Wissensdifferenzen und Wissensschieflagen dar und fragen nach dem Nutzen und Un-Nutzen bestimmter Wissenszusammenhänge. Doch auch formal-ästhetisch inszeniert der Text Unnützes Wissen, indem er eine regelrechte Ästhetik der Irrelevanz kreiert.

\section{III.1 Unnützes Wissen als Textstruktur}

Bereits die strukturelle Komposition des Romans baut auf einer Abfolge scheinbar Unnützen Wissens auf. Insgesamt folgt der Roman chronologisch den drei Auszügen von Don Quixote und Sancho Pansa, die sich wiederum zergliedern in eine sich fast endlos wiederholende Redundanz immer ähnlicher Abenteuer. Zwischenzeitlich fragt man sich als Leser, ob einige Kapitel für den Gesamtzusammenhang nicht eigentlich entbehrlich und daher unnütz sind. Manche Abenteuer zeichnen sich durch eine derart gesteigerte Absurdität, Skurrilität und Surrealität aus, dass sie eigentlich aus dem Gesamtrahmen herausfallen. ${ }^{15}$ Darüber hinaus wird die repetitive Abenteuerstruktur durch eingestreute Theaterstücke, Schäferspielepisoden, Briefe,

14 "Nachdem er [Don Quixote] alles vollbracht hatte, wollte er gleich die Trefflichkeit seines erfundenen köstlichen Balsams probieren, er trank also das Übriggebliebene aus, was er nicht in die Ölflasche hatte füllen können [...]. Er hatte es aber kaum getrunken, als ihn ein so heftiges Erbrechen befiel, daß er nichts im Magen behielt [...]. Sancho Pansa [...] bat um das, was noch im Topfe zurückgeblieben sei [...]. Indem fing der Balsam an zu wirken und der arme Stallmeister entledigte sich seiner Bürde aus beiden Kanälen, daß weder die Binsenmatte, auf der er lag, noch das Tuch, mit dem er zugedeckt war, jemals wieder gebraucht werden konnten" (DQ127f.).

15 Man denke hier z.B. an Episoden, wie Don Quixotes Erlebnis in der Höhle von Montesinos oder Sanchos tatsächlich eintretende Statthalterschaft einer Insel, bei der er sich plötzlich wie ein weiser Herrscher gebärdet. 
Empfehlungsgedichte, Sonette und insbesondere novellenartige Binnengeschichten unterbrochen, die von Figuren und Geschehnissen erzählen, die zunächst scheinbar nichts mit der Haupthandlung zu tun haben. ${ }^{16}$ Zwar bestehen gewisse motivische Zusammenhänge zwischen diesen Einsprengseln (Entremes) untereinander sowie mit der Haupthandlung, »[k]ritisch muss man jedoch fragen, ob vergleichbare Themen und Motive der Novellen im ersten und im zweiten Teil schon die Konzeption des Werkes als Ganzheit beweisen. ${ }^{17}$ Vor allem das heterogene Nebeneinander völlig unterschiedlicher Texttypen und Genrezitaten mutet als ein überflüssiges Durcheinander an, dessen Sinn sich zunächst kaum erschließt. ${ }^{18}$

\section{III.2 Erzählend vom unnützen Erzählen erzählen: Erzählerische Performativität des Unnützen}

Auch die Erzählinstanz unterbricht ihre Erzählung wiederholte Male, um dem Leser Wissen über ihren eigenen Status und den der erzählten Geschichte mitzuteilen, das für den Fortgang der Handlung kaum relevant ist, unterbrechend und digressiv wirkt und teilweise von der Erzählinstanz selbst als unnütz eingestuft wird. So wird beispielsweise am Ende des oft zitierten achten Kapitels plötzlich im Erzählen innegehalten und von mehreren angeblichen Überlieferungsschichten und unterschiedlichen Quellenlagen der Geschichte des Don Quixote berichtet. Auch werden mehrere Erzähler inszeniert, die sich im Verlauf der Geschichte zu einer multiplen Erzählinstanz verdichten. Diese setzt sich aus erstem und zweitem Erzähler, einem arabischen Historienschreiber namens Cide Hamete Benengeli und dessen Übersetzer zusammen. Der erste Erzähler wird nach acht Kapiteln von einem zweiten Erzähler abgelöst, der dies auch thematisiert:

16 Verwirrung stellt sich ganz besonders gleich zu Beginn des Romans ein, wenn man als Leser mit einer Reihe von Lobgedichten konfrontiert ist, deren Sinn sich frühestens nach der Lektüre des Romans erschließt.

17 Christoph Strosetzki, Miguel de Cervantes. Epoche-Werk-Wirkung, München 1991, S. 137.

18 Diese Heterogenität von Gattungen, Genres sowie die scheinbar auseinanderfallende Vielheit von Themen und Motiven wurden vor allem von den deutschen Romantikern als Stärke des Romans verstanden. Sie lassen sich insbesondere im Zusammenhang mit der Schlegel'schen sprogressiven Universalpoesier sehen. Vgl. Sebastian Neumeister, Der romantische Don Quijote, in: Christoph Strosetzki (Hg.), Miguel de Cervantes' Don Quijote, S. 301-314. 
Schade aber ist es, daß gerade bei dieser Stelle der Autor abbricht [...], mit der Entschuldigung [...], daß er nichts Weiteres von Don Quixotes Taten vorgefunden, als was er bereits erzählt habe. Der zweite Autor [also der jetzige Erzähler] konnte aber unmöglich glauben, daß eine so treffliche Geschichte so ganz der Vergangenheit sollte überliefert sein oder daß die herrlichen Köpfe in la Mancha so wenig Wißbegier haben sollten, daß sich nicht noch in den Archiven oder in einigen Schreibpulten Papiere vorfinden dürften, die von diesem berühmten Ritter Meldung tun. Diesen Gedanken nährte ich und hoffte demnach, den Schluß dieser anmutigen Historie anzutreffen, welches mir auch unter den Begünstigungen des Himmels [...] gelungen ist $[\ldots]$. (DQ70)

Im Folgenden berichtet dieser zweite Erzähler, er habe auf einem Markt in Toledo zufällig von einem Seidenhändler eine weitere Überlieferung des Lebens des Don Quixote erworben, die eben von jenem arabischen Historienschreiber namens Cide Hamete Benengeli verfasst worden sein soll. Der zweite Erzähler fungiert damit als fiktive Herausgeberfigur, "[...] die aus unterschiedlichen Materialien ein Buch zusammenstellt « ${ }^{19}$ - wodurch sich innerhalb des fiktionalen Universums auch die Kapitelüberschriften erklären. Den auf dem Markt erstandenen Text betreffend, gibt der Erzähler selbst zu, dass nicht alle darin geschilderten Geschehnisse mit seiner ersten Quelle übereinstimmen, was jedoch nicht weiter wichtig sei: "Ich könnte noch einige andere Abweichungen anführen, aber sie sind alle unwichtig und keine tut der Wahrheit der Geschichtserzählung Eintrag (DQ73). Er disqualifiziert das Wissen, das er dem Leser mitteilt, während er es mitteilt, als wenig zur Sache tuend und markiert es damit als eigentlich unwichtig. Gleichzeitig unterstellt er dem Text des arabischen Historienschreibers Cide Hamete einen Mangel an Glaubwürdigkeit, da es - seiner Meinung nach - »dieser Nation eigentümlich sei, zu lügen« (DQ73). Weshalb er all diese Informationen an den Leser weiterreicht, wird nicht erklärt, und es stellt sich die Frage nach deren weiterem Nutzen, denn zunächst entsteht durch dieses Erzählexperiment mit »different registers and [...] some highly original shifts of narrative voice $[\ldots]$ a certain amount of confusion ${ }^{20}{ }^{20}$

\section{III.3 Metafiktion als Unnützes Wissen des Textes über sich selbst?}

Der Gedanke des Unnützen Wissens und der Verunsicherung von Wissen spielt sich auch auf der Meta-Ebene des Textes ab, auf der sich der Text so-

19 Christoph Strosetzki, Miguel de Cervantes, S. 132.

20 James A. Parr, Don Quixote, Don Juan, and Related Subjects. Form and Tradition in Spanish Literature, 1330-1630, Selinsgrove PA 2004, S. 36. 
zusagen selbst als fiktionaler Text zum Wissen wird, indem er sich selbstreferentiell als solchen erkennt, beschreibt und reflektiert. Wissen überspringt dabei - teils gegen die Erzähllogik - diegetische Ebenen und damit unterschiedliche Realitätsniveaus des Textes. So beispielsweise, wenn sich in Don Quixotes Bibliothek der Schäferroman La Galatea von Cervantes selbst findet ${ }^{21}$ oder erzählte Figuren aus der intradiegetischen Ebene als handelnde Figuren auf der extradiegetischen Ebene auftauchen. Vor allem der zweite Teil des Romans ist hochgradig selbstreferentiell; er reflektiert und kommentiert über viele Seiten hinweg den ersten Teil. Beispielsweise wird kommentiert, dass Sanchos Esel plötzlich wieder ohne weitere Erklärung aufgetaucht ist, wo er doch eigentlich verschwunden gewesen war. ${ }^{22}$ Dies ist eingebettet in die prominenteste Metalepse des Romans, nämlich die Tatsache, dass Don Quixote und Sancho im zweiten Teil von der Existenz eines Buches Don Quixote, verfasst von einem gewissen Cide Hamete Benengeli, erfahren, in dem von ihren Abenteuern berichtet wird. Dieses Wissen und der Gedanke an einen möglichen zweiten Teil wird zur Motivation eines erneuten Ausritts: "Der Herr Maure [Cide Hamete] oder was er sonst sein mag, sehe doch ja zu, was er tut; denn ich und mein Herr wollen ihm so viel Stoff zu Abenteuern und mancherlei Begebenheiten in die Hände arbeiten, daß er nicht nur den zweiten Teil, sondern wohl den hundertsten schreiben kann« (DQ 520). Auch erfahren sie etwas später von einem zirkulierenden, unautorisierten zweiten Teil, der von ihren erneuten Ausritten berichtet und der nicht von Cide Hamete stammt - was bei den beiden Hauptfiguren den Wunsch auslöst, zu beweisen, dass der plagiierende Autor von einem sfalschen Don Quixote erzählt: "[...] so soll die ganze Welt die Lügen dieses neuen Geschichtsschreibers erkennen, damit die Leute einsehen, wie ich nicht der Don Quixote bin, von dem er erzählt" (DQ 914). Es wird dadurch innerfiktional Bezug auf ein in der außerfiktionalen Realität existierendes Plagiat des Don Quixote genommen: das von Alonso Fernández de Avellaneda, das auf die Erfolgswelle von Cervantes' Roman aufsprang - was Cervantes nicht wenig verärgerte. Möglicherweise ist dies auch ein Grund, weshalb der Protagonist im cervantinischen, 1615 erschienenen, zweiten Teil des Don Quixote sterben muss - just um eine weitere ungewollte Fortsetzung der Geschichte zu verhindern. Cervantes

21 Vgl. DQ, Teil I, Kapitel 6, S. 56.

22 "[...] er [Cide Hamete] hat zu erzählen vergessen, wer der Räuber war, der dem Sancho seinen Grauen stahl; denn dieses wird doch nicht deutlich, sondern man findet nur geschrieben, daß er ihm gestohlen wurde, und bald darauf finden wir ihn auf seinem eigenen Esel wieder beritten, ohne daß dieser zum Vorschein gekommen« (DQ 517). 
schließt dadurch das fiktionale Universum mit der Realität des Lesers und seiner eigenen Realität kurz. Doch ist diese metafiktionale Struktur für den Text snützlich`?

\section{Inversbewegung}

Es zeigt sich also auf allen Ebenen des Textes, dass literarisches Wissen vordergründig als relativ unnütz markiert wird, sei es durch die Stigmatisierung literarischen Wissens als Wahn in der Figur des Don Quixote, dessen unpraktischen und teils lebensgefährdenden Versuch der Übertragung dieses Wissens auf die Lebenswelt oder durch eine formalästhetisch inszenierte Irrelevanz, die stets an die Idee der Literatur zurückgebunden ist. Gleichzeitig wird jedoch das, was vordergründig als unnütz und überflüssig erscheint, durch eine Inversbewegung des Textes wieder eingeholt und ins Gegenteil gewendet, wodurch eine Doppelbewegung des Textes entsteht. Auf der Inhaltsebene zeigt sich dies etwa in dem Kapitel mit dem Titel »Lustiger und feierlicher Gerichtstag, den der Pfarrer und Barbier im Büchersaale unseres scharfsinnigen Edlen hielten« (DQ 51): Don Quixotes Wahn wird ja von seiner übermäßigen Lektüre von Ritterromanen ausgelöst und äußert sich auch insbesondere dann, wenn die Situation an dieses Genre rührt. Die ihm nahestehenden Personen - Nichte, Hausmädchen, Pfarrer und Barbier - wollen aus diesem Grunde seine Bibliothek vernichten, denn die "verfluchten Ritterbücher, die er immer las, [haben ihm] den Verstand verrückt« (DQ49). Es wird der Beschluss gefasst, alle Bücher aus Don Quixotes Besitz in inquisitorischer Geste aus dem Fenster zu werfen und auf einem Scheiterhaufen zu verbrennen. Zunächst werden die Bücher also nicht nur als aussortierenswert und nutzlos, sondern geradezu als gefährlich beurteilt. Doch bei genauerer Betrachtung der Bücher werden Pfarrer und Barbier immer zögerlicher beim Aussortieren und beginnen, die Werke nach Maßstäben der Literarizität und Ästhetik zu sortieren und bestimmte Exemplare vor dem Feuer zu retten und gar mit nach Hause zu nehmen:

»Bei meinem heiligen Amte«, erwiderte der Pfarrer, »seit Apollo Apollo gewesen, die Musen Musen und Poeten Poeten, ist kein so anmutiges und tolles Buch als dieses geschrieben, es ist das trefflichste, ja das einzige unter allen, die in dieser Gattung jemals an das Licht der Welt getreten sind, und wer es nicht gelesen hat, kann überzeugt sein, daß er noch nichts vollkommen Schönes gelesen hat. Gebt es gleich her, 
Gevatter, dieser Fund ist mir mehr wert, als wenn mir einer ein Priesterkleid von dem groben florentinischen Tuche geschenkt hätte.« (DQ 56) ${ }^{23}$

Der Literatur wird also doch ein Wert beigemessen, selbst wenn es snur ein ästhetischer ist. Darüber hinaus wird auch die Stigmatisierung des Don Quixote als Wahnsinniger entschärft. Zwar verfällt er stets in Wahn, wenn es um das Thema der Ritterschaft geht, doch erscheint er "in allen übrigen Dingen [...] gescheit und verständig“ (DQ361), spricht durchaus wohl reflektiert und rhetorisch geschliffen, sodass andere Figuren seinen Ausführungen gerne und aufmerksam Gehör schenken: "Auf diese Art und in so guten Ausdrücken, fuhr Don Quixote in seiner Rede fort, so daß ihn keiner von seinen Zuhörern für einen Toren halten konnte [...] Der Pfarrer sagte, daß er sehr recht in allem habe, was er zugunsten der Waffen behauptet, und daß er selber, obgleich Gelehrter und Graduierter, derselben Meinung sei« (DQ 357 u. 361). ${ }^{24}$ Durch diese Differenzierung bezüglich des Wahnsinns von Don Quixote wird in gleichem Zuge literarisches Wissen aus dem Kontext der wahnhaften Verirrung befreit, denn auch hier wird unterschieden: Sofern es sich nicht um Ritterromane handelt, versteht Quixote das Spiegelverhältnis von Realität und Fiktion durchaus. Er erscheint als gebildeter Literaturkenner, der in äußerst scharfsinniger und differenzierter Weise literarisches Wissen und reale Umstände aufeinander beziehen kann - in seinen Reflexionen zeigen sich denn auch die Spuren nicht nur der zeitgenössischen Literatur, sondern auch klassischer Schriften der Poetik und Ästhetik (Aristoteles, Horaz, Platon, etc.). Vor allem in seinen Ausführungen zur Komödie ist das erkennbar:

[...] es kann sein, daß der Schmuck der Komödie nicht echt war; er war vielleicht unecht und nur zum Schein, wie es die Komödie selber ist, welche du, Sancho, hochschätzen und in deine Gunst aufnehmen sollst, und aus dem nämlichen Grunde auch diejenigen, die sie vorstellen, wie die, welche sie schreiben. Denn alle sind Mittel dazu, im Staate etwas sehr Gutes hervorzubringen, indem sie uns bei jedem Schritte einen Spiegel vorhalten, in welchem man die Handlungen der Menschen wahrnehmen kann, und keinen anderen Vergleich gibt es, der uns so lebendig vor die Augen stellt, was wir sind und was wir sein werden, als die Komödie und die

23 Vgl. ebenso: "Diese drei Bücher«, sagte der Pfarrer, ssind die besten heroischen Gedichte, die in kastilianischer Sprache geschrieben sind, sie können sich mit den berühmtesten der Italiener messen, hebt sie als die köstlichsten Stücke der Poesie auf, die Spanien besitzt« (DQ57).

24 Es handelt sich hier um die Rede über Waffen und Wissenschaften, die Don Quixote im 37. und 38. Kapitel des ersten Teils hält. Auch spricht er mit »einer angenehmen und sanften Stimme« (DQ361). 
Komödianten. Hast du nämlich nicht schon eine Komödie vorstellen sehen, in welcher Könige auftreten, Kaiser und Päpste, Ritter, Damen und verschiedene andere Personen? [...] und wenn die Komödie zu Ende ist und sie ihre Kleider ausziehen, sind sich alle Schauspieler gleich. [...] Ebenso [...] geht es in der Komödie und dem Schauspiel dieser Welt, wo etliche Kaiser spielen, andere Päpste, und kurz, ebenso viele Rollen, als nur in der Komödie vorkommen können; wenn es aber zu Ende ist, wenn das Leben nämlich aus ist, zieht der Tod allen die Kleider aus, nach welchen sie sich unterschieden, und in ihren Gräbern sind sie gleich. (DQ569f.)

In seiner rhetorischen Gewandtheit kleidet er die Darstellung dieses Spiegelverhältnisses in einen Vergleich, der in einer mise en abyme und auf den barocken Topos des theatrum mundi anspielend, Fiktion und Wirklichkeit als strukturgleich darstellt: Das Schauspiel zeugt von der Welt und die Welt ist nichts anderes als ein Schauspiel. In dieser mimetischen Beziehung zwischen Fiktion und Wirklichkeit wird Ersterer über ein delectare hinaus auch die Funktion eines prodesse zugesprochen; durch sie können wir uns selbst erkennen, lernen und unsere Wirklichkeit besser gestalten. ${ }^{25}$ Die Übertragung literarischen Wissens auf die Lebenswelt kann so wahnsinnig also nicht sein.

Auch das auf so vielschichtige Weise inszenierte Wissen des Textes um sich selbst wird vom Unnützen ins Nützliche geholt. Zum einen ist dieses scheinbar unnötige, weil diegetisch-unlogische Wissen als Spiel der Literatur mit sich selbst äußerst amüsant und besitzt damit bereits einen Unterhaltungswert, nach dessen Nutzen nicht weiter gefragt werden muss. Zum anderen macht sich der Text durch dieses metafiktionale Spiel auch, vor allem im zweiten Teil, zu einem literarhistorisch interessanten Zeugnis der Literaturrezeption - was einerseits die Rezeptionshaltung von Cervantes' angeht, andererseits aber auch die Aufnahme seines Romans beim zeitgenössischen Publikum, die er wiederum in den zweiten Teil einarbeitet: "Cervantes incorporates criticism and the aesthetics of reception into the second part in an intriguing way, since they now refer to his own narrative. ${ }^{26}$ Darüber hinaus besitzen diese Kommentare eine nicht zu vernachlässigende Funktion auf der Plot-Ebene. Vor allem das Wissen der Figuren um die Existenz eines Buches über ihre eigenen Erlebnisse, ihr Gedanke an eine niedergeschriebene Fortführung der Abenteuer sowie die darin sich spiegelnden Reaktionen

25 Dieses Spiegelverhältnis wird durch den in dieser Episode erscheinenden »mutigen Ritter von den Spiegeln« (DQ 569) personifiziert.

${ }^{26}$ Edward H. Friedman, Books Errant: The Objects of Invention in Don Quixote, in: Harald Bloom (Hg.), Miguel de Cervantes's Don Quixote. Blooms Modern Critical Interpretations, New York 2010, S. 43-56, hier S. 53. 
des realen Publikums auf den ersten Teil fungieren als wichtige Motivationsimpulse für entscheidende Handlungsaspekte des Textes - vor allem im dritten und letzten Auszug der beiden Hauptfiguren: »Furthermore, reader-response becomes the catalyst or motivating factor-the basic apparatus of the plot-in Part $2 \varkappa_{.}{ }^{27}$ Auch eine genauere Betrachtung der disparaten Handlungsbewegungen und eingestreuten Binnengeschichten, Theaterstücke etc. führt dazu, »daß der Eindruck eines Versuches entsteht, durch das Spiel mit Variationen neue Lösungen einer komplexen Materie zu ergründen ${ }^{28}{ }^{28}$ In diesem Sinne lässt sich ebenfalls die komplexe und verwirrende Erzählinstanz interpretieren, die ihr Spiel mit Authentizität und Authentizitätsfiktion treibt: Denn wird nicht dadurch die Einsicht vermittelt, dass es sich bei dem Phänomen Wirklichkeit, das dem Protagonisten so große Probleme bereitet, um eine Größe handelt, die abhängig ist vom Betrachter und dadurch nur adäquat durch eine multiperspektivisch gestaltete Erzählweise wiedergegeben werden kann? Wird nicht dadurch formal-ästhetisch das Hauptthema des Romans, nämlich die mangelnde Trennschärfe von Realität und Fiktion, gespiegelt?

Wenn all die zunächst irrelevant und überflüssig erscheinenden Momente des Textes letztlich also doch eine funktionale Eigenschaft besitzen, wozu dann dieses Verweben des literarischen Wissens mit der Idee des `Unnützen«? Die Antwort darauf liegt in der Natur des Textes als Parodie.

\section{Die Geburt des Romans aus der Parodie}

Der Text stellt auf allen Ebenen eine Parodie dar, eine Parodie ziemlich aller literarischen Gattungen und "pre-novelistic «" ${ }^{29}$ Genres, insbesondere der zu Cervantes' Zeit so beliebten Ritterromane. Dafür steht die Figur des

27 Ebd.

28 Christoph Strosetzki, Miguel de Cervantes, S. 136.

29 Anthony J. Cascardi, Don Quixote and the Invention of the Novel, in: Cascardi (Hg.), The Cambridge Companion to Cervantes S. 58-79, hier S. 58. Der Roman stellt eine Fülle von Referenzen auf vielerlei Gattungen und Genres dar, v.a. auf die Pastorale im Stile des kastilischen Schäferromans, wie etwa La Diana eines Jorge de Montemayors oder auch Cervantes' eigenen Schäferroman La Galatea (besonders in den Kapiteln zur Schäferin Marcella und ihrem Verehrer bzw. Verfolger Grisóstomo, Teil I, Kapitel 11-14); auf die fingierte Autobiographie, v.a. in Form des Pikaroromans im Stile des anonym erschienenen Lazarillo de Tormes und des Guzmán de Alfarache von Mateo Alemán; auf die italienische Epik der Renaissance, wie dem Orlando furioso von Ludovico Ariosto; auf den Ritterroman, v.a. Amadís de Gaula (wie in der Fassung von Garci Rodríguez de Montalvo); etc. 
Don Quixote topisch ein. So wie Don Quixote auszieht und nachahmendaktionistisch Rittergeschichten imitiert, so zieht auch Cervantes' Text aus und imitiert sie textuell. Parodien beziehen sich auf vorhergehende Texte und Textarten, deren Inhalte und Schreibpraktiken, Techniken und Konventionen. Sie machen es sich zur Aufgabe, durch Verspottung einen buchstäblichen »Gegengesang « ${ }^{30}$ anzustimmen. In verlachender Gebärde bringen sie sich in reflektierende Distanz zu ihren Intertexten, die dadurch zunächst kritisiert, ironisiert und eben auch als überkommen suspendiert werden. Jedoch ist jede Parodie gleichzeitig fundamental ihrem Gegenstand des Spotts verpflichtet, da sie in ihrem Sein genuin von ihm abhängig ist; nur durch ihn und in Bezug auf ihn kommt sie zur Existenz. Parodieren bedeutet nicht zwingend eine negative Auseinandersetzung mit anderen Texten - wie es sich im Don Quixote schon allein in der Tatsache zeigt, dass in erster Linie schlechte Ritterromane parodiert werden, ohne aber das Genre generell vollkommen abzuwerten. ${ }^{31}$ Die Parodie stellt daher, vor allem im vorliegenden Falle, nicht nur eine destruierende Bewegung, ${ }^{32}$ sondern auch einen "alternative[n] 'Nebengesang " und gar ein produktives Wieder-Aufnehmen, ein "abgewandeltes Wiederholen von überlieferten Texten als wichtiges Grundmuster der literarischen Entwicklung « dar. ${ }^{33}$ Damit bedeutet Parodieren auch Bewahrung und Rettung von literarischem Wissen durch das Medium der Literatur selbst. Die Parodie, als Oszillieren zwischen `Gegengesang und produktivem Wiederanstimmen, stellt so einen synthetischen Doppeldiskurs dar, in den sich die oben geschilderte Doppelbewegung des Unnützen im Don Quixote einschreibt. Dazu soll ein letztes Beispiel aus dem

${ }^{30}$ Lemma >Parodiec, in: Ansgar Nünning (Hg.), Metzler Lexikon. Literatur- und Kulturtheorie. Ansätze - Personen - Grundbegriffe, 2., überarb. u. erw. Aufl., Stuttgart/Weimar 2001, S. 492.

31 Beispielsweise wird in der Szene, in der Don Quixotes Bibliothek verbrannt wird, vom Barbier ein Unterschied zwischen schlechten und guten Ritterromanen wie dem Amadis gemacht: "'[...] man hat mir auch gesagt, daß dies Buch das beste von allen in dieser Gattung sei, und darum könnte man ihm wohl als den einzigen seiner Gilde vergeben« (DQ51).

32 Vgl. Hans-Jörg Neuschäfer, Der Sinn der Parodie im Don Quijote, Heidelberg 1963, S. 10: "[...] die Parodie des Quïjote - wie überhaupt jede echte Parodie - [darf] nicht als eine bloße Zerstörung des Parodierten verstanden werden [...], sondern vielmehr als eine Auflösung, in der die epische Welt als ein Aspekt der romanhaften (d.h. hier als Phantasie des Landjunkers) noch immer erhalten bleibt, so daß der Quijote nicht nur als Antithese gegen den Ritterroman, sondern auch als Synthese gesehen werden kann, durch die der Widerspruch zwischen beiden Gattungen [Roman und Epos] sogleich auch wieder aufgehoben ist.".

33 'Parodie‘, in: Ansgar Nünning (Hg.), Metzler Lexikon. Literatur- und Kulturtheorie, S. 492. 
Text aufgerufen werden. Im Prolog wird ein Gespräch zwischen fiktivem Autor und einem seiner Freunde inszeniert. Der fiktionale Autor drückt seine Befangenheit über die Legitimität seines Buches aus, da es angeblich aller intertextuellen Referenzen entbehre, durch die sich gute bzw. gelehrte Literatur auszeichne:

Soll ich denn nun nicht darüber in Sorgen sein, was der alte Gesetzgeber, der Haufen genannt, sagen wird, wenn er nun sieht, wie [...] ich mit einer Lektüre hervortrete [...] gänzlich ohne Gelehrsamkeit und Literatur, ohne Bemerkungen am Rande und ohne Anmerkungen am Ende des Buches, wie ich doch sehe, dass andere Bücher eingerichtet sind, auch fabelhafte und weltliche, die voller Sentenzen des Aristoteles, Plato und der ganzen Schar der Philosophen stecken, worüber sich alsdann die Leser verwundern und die Verfasser für belesene, gelehrte und beredte Männer halten! [...] Alles dieses mangelt meinem Buche, denn ich habe am Rande nichts bemerkt und am Ende nichts angemerkt, noch weniger weiß ich, welchem Autor ich folge [...]. (DQ8)

Sein Freund rät ihm daraufhin, kurzerhand einen Schein der gelehrsam wirkenden intertextuellen Referenzen zu entwerfen, da diese doch eigentlich ohnehin unnütz, weil dem Publikum egal, seien:

Jetzt bleibt uns nur noch die Zitation der Autoren übrig, die man in anderen Büchern findet und die in den Eurigen fehlen. Diesem abzuhelfen gibt es ein sehr bequemes Mittel, denn Ihr braucht nur eins von den Büchern zu nehmen, in denen sie alle, wir Ihr sagt, von $\mathrm{A}$ bis $\mathrm{Z}$ zitiert sind. Das nämliche Abc könnt Ihr nun auch Eurem Buche anheften: sieht man auch die Lüge ganz deutlich, so tut Euch das nichts, da Ihr alle die Autoren nicht braucht, und vielleicht ist doch einer oder der andere so einfältig, dass er glaubt, Ihr hättet sie wirklich alle bei Eurer einfachen schlichten Erzählung gebraucht. Überdies wird es niemand untersuchen, ob Ihr ihnen gefolgt seid oder nicht, denn keiner kümmert sich darum. Ihr habt aber [...] aller der Sachen nicht nötig [...]. Euer Hauptzweck ist, das darzustellen, was Ihr schreiben wollt, und je mehr Ihr dies erreicht, je vorzüglicher wird Euer Buch sein, [...] Euer Augenmerk ist, Eure Erzählung in einem einfachen, ausdrucksvollen, edlen und geziemenden Stil zu verfassen, dass Eure Perioden sich wohlklingend und anständig fortbewegen und dass Ihr nach Eurer Absicht alles deutlich darstellt, ohne Eure Ideen durch Spitzfindigkeit oder Dunkelheit zu verwirren. (DQ12)

Was letztlich zähle, sei die Geschichte ohne Schnörkel, die keine Legitimierung durch intertextuelle Einbettung benötige. Einerseits wird so bereits im Vorwort literarisches Wissen und literarische Tradition vordergründig als unnützer Ballast markiert - es wird in emanzipatorischer Gebärde scheinbar mit dem rhetorischen Konzept der imitatio gebrochen. Andererseits signalisiert der Text gleichzeitig, dass dies nicht möglich ist und dass jeder Text in einem literarischen Kontext steht - der Gedanke der imitatio (in allen ihren 
möglichen Formen) und der mimesis (wenn auch in verballhornter Version) bildet ja auch die Grundlage von Don Quixote. Natürlich handelt es sich bei diesem Spiel um eine inszenierte Koketterie, denn Cervantes und sein Text sind - wie sich von Beginn an zeigt - mit allen Wassern literarischen Wissens gewaschen. ${ }^{34}$ Durch das absurd-sinnlos anmutende Spiel mit angeblich überflüssigem literarischen Wissen lotet der Text seinen Status als Text und mithin seine eigenen erzählerischen Möglichkeiten aus. Dadurch wird sich nicht nur das Buch selbst zum Wissen, sondern es macht sich literarisches Wissen zum Wissen, mit dem es zu jonglieren beginnt. Es wird also nicht, wie es zunächst scheinen könnte, eine Poetologie des unnützen literarischen Wissens entworfen, sondern eine der Parodie verpflichtete diskursive Doppelbewegung zur Poetologie erhoben, die letztlich ihr schöpferisches Potential zur literarischen Weiterentwicklung bzw. Innovation eröffnet. Denn Don Quixote konstituiert in dieser Doppelbewegung den europäischen Roman als Gattung und gebiert sich als solcher - aus der Literatur und im Medium der Literatur - selbst: "True dialectical parody can lead [...] to a synthesis of foreground and background that in time transcends its roots and inaugurates a new autonomous form. [...] the parody of the romances of chivalry gave us Don Quijote, and with it the novel as we know it today. « ${ }^{35}$

Literarisches Wissen im Don Quixote ist daher alles andere als unnütz. Im Gegenteil, es führt zu spielerischem Experiment und dadurch zu literarischer Innovation. Es handelt sich also, wenn man so will, um eine `Don Quixoterier im positiven Sinne.

34 Bereits das Eingangsgespräch zwischen fiktivem Autor und seinem Freund ist als Anspielung auf die sokratischen Dialoge zu verstehen.

35 Linda Hutcheon, Parody Without Ridicule: Observations on Modern Literary Parody, in: Canadian Review of Comparative Literature 5/2 (1978), S. 201-211, hier S. 210. Ebenso: Anthony J. Cascardi, Don Quixote and the Invention of the Novel, S. 64: »As for Cervantes, his goal was not so much to reproduce the style or form of the books of chivalry as to allow them to be spoken again, but undercut by irony, and in a mock-heroic way. The same holds true for the genres of the pastoral and the picaresque, and for all of the others already mentioned. They are all transformed in the Quixote by their displacement in time and space and by their situation relative to other discourses undergoing a similar process of rearticulation. [...] And so Cervantes sinvents< the novel by moving the discourses of pastoral, chivalric, picaresque, etc., from their original locations in culture and into the domain of self-conscious parody and pastiche«. 
